\title{
THERMAL-NEUTRON CAPTURE GAMMA RAYS FROM NATURAL MAGNESIUM AND ENRICHED ${ }^{25} \mathrm{Mg}$
}

\author{
P. SPILLING, H. GRUPPELAAR and A. M. F. OP DEN KAMP
}

Fysisch Laboratorium der Rijksuniversiteit, Utrecht, the Netherlands

\author{
Received 1 June 1967
} \begin{abstract}
could be fitted into the level schemes of ${ }^{25} \mathrm{Mg},{ }^{26} \mathrm{Mg}$ and ${ }^{27} \mathrm{Mg}$.
The $Q$-values for the following reactions have been determined

$\begin{array}{ll}{ }^{24} \mathrm{Mg}(\mathrm{n}, \gamma)^{25} \mathrm{Mg} & Q=7332.8 \pm 0.4 \mathrm{keV}, \\ { }^{25} \mathrm{Mg}(\mathrm{n}, \gamma)^{26} \mathrm{Mg} & Q=11096.0 \pm 0.5 \mathrm{keV}, \\ { }^{26} \mathrm{Mg}(\mathrm{n}, \gamma)^{27} \mathrm{Mg} & Q=6446.3 \pm 0.9 \mathrm{keV}, \\ { }^{56} \mathrm{Fe}(\mathrm{n}, \gamma)^{57} \mathrm{Fe} & Q=7643.9 \pm 1.5 \mathrm{keV}, \\ { }^{83} \mathrm{Cu}(\mathrm{n}, \gamma)^{64} \mathrm{Cu} & Q=7916.5 \pm 1.5 \mathrm{keV}, \\ { }^{207} \mathrm{~Pb}(\mathrm{n}, \gamma)^{208} \mathrm{~Pb} & Q=7371.0 \pm 2.5 \mathrm{keV} .\end{array}$
\end{abstract}

Abstract: Gamma rays from neutron capture in natural magnesium and in enriched ${ }^{25} \mathrm{Mg}$ have been studied with $\mathrm{Ge}(\mathrm{Li})$ detectors. Altogether $101 \gamma$-rays have been observed. Most of these $\gamma$-rays

If it is assumed that the capture cross section for natural magnesium amounts to $64 \pm 3 \mathrm{mb}$, the capture cross sections for ${ }^{24} \mathrm{Mg},{ }^{25} \mathrm{Mg}$ and ${ }^{26} \mathrm{Mg}$ have been determined as $52 \pm 13 \mathrm{mb}, 182 \pm 50$ $\mathrm{mb}$ and $34 \pm 10 \mathrm{mb}$, respectively.

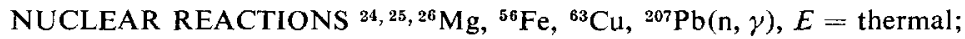
measured $\sigma\left(E_{\gamma}\right)$; deduced $Q$.



\section{Introduction}

The ${ }^{25} \mathrm{Mg}(\mathrm{n}, \gamma)^{26} \mathrm{Mg}$ reaction has been investigated very little. Yet this reaction may give valuable information on the ${ }^{26} \mathrm{Mg}$ level scheme.

Preliminary measurements of $\gamma-\gamma$ coincidence spectra and angular correlations with $\mathrm{NaI}$ detectors made it clear that the decay is quite complicated. Several sets of levels, situated very close together, are excited. It was felt that much better resolution was needed in order to clarify the situation.

The present paper describes an investigation of the $\gamma$-ray spectra from thermalneutron capture in natural magnesium and in magnesium enriched in ${ }^{25} \mathrm{Mg}$ by means of $\mathrm{Ge}(\mathrm{Li})$ counters.

Prior to the present investigation, the gamma rays from neutron capture in natural magnesium had been studied with a pair spectrometer ${ }^{1}$ ) and a magnetic Compton spectrometer ${ }^{2}$ ). Altogether 27 lines had been found. Most of these lines were fitted into the level schemes of ${ }^{25} \mathrm{Mg},{ }^{26} \mathrm{Mg}$ and ${ }^{27} \mathrm{Mg}$, but not all of them in a satisfactory way. Several weak high-energy lines were indicated, which were not observed in the 
present investigation. Most of the data known for the three magnesium isotopes are tabulated in the review article by Endt and van der Leun ${ }^{3}$ ).

The nucleus ${ }^{26} \mathrm{Mg}$ is rather difficult to investigate because of the many doublets and triplets appearing in the level scheme. Partly because of this, $l_{\mathrm{n}}$ value assignments from the $(d, p)$ reaction are made difficult. Also it is found that for many levels, more than one $l_{\mathrm{n}}$ value contribute to the transitions ${ }^{4}$ ). Therefore many of the $l_{\mathrm{n}}$ values are doubtful.

By combining the results of investigations of the $(t, p),(d, p)$ and $\left(d, d^{\prime}\right)$ reactions leading to the levels in ${ }^{26} \mathrm{Mg}$, Hinds $\epsilon$ t al ${ }^{5}$ ) were able to assign spins and parities to levels up to $7 \mathrm{MeV}$. Strikingly, no negative parity state was found below $6.8 \mathrm{MeV}$.

The triplet at $E_{\mathrm{x}}=4.3 \mathrm{MeV}$ was not completely resolved in the (t, p) and (d, p) reactions, and therefore the spin assignments to these levels were difficult. However, it was shown that the upper level in this triplet certainly has $J^{\pi}=2^{+}$. For the other two levels no unique assignments could be made. From systematics of doubly even nuclei in the neighbourhood of ${ }^{26} \mathrm{Mg}$, one would expect a $4^{+}$level between 4 and 5 $\mathrm{MeV}$. Thus one of the lower levels in the triplet might well have $J^{\pi}=4^{+}$.

\section{Experimental arrangement}

As samples, $6 \mathrm{~g}$ of natural magnesium metal and $2,4 \mathrm{~g} \mathrm{MgO}$ enriched to $85 \%$ in ${ }^{25} \mathrm{Mg}$ were used. The enriched $\mathrm{MgO}$ was on loan from the Electromagnetic Separation Group at Harwell, England. It was placed in a thin-walled teflon container.

The samples were placed in the neutron beam emerging from one of the radial beam holes in the Dutch High Flux reactor in Petten. The thermal-neutron flux at the sample was approximately $10^{7} \mathrm{~cm}^{-2} \cdot \mathrm{s}^{-1}$. At the position of the sample, the neutron beam was surrounded by a ${ }^{6} \mathrm{LiF}$ shield, absorbing scattered neutrons.

For $\gamma$-ray detection, an Ortec $1.6 \mathrm{~cm}^{3} \mathrm{Ge}(\mathrm{Li})$ detector, $4 \mathrm{~mm}$ thick and a RCA $5 \mathrm{~cm}^{3} \mathrm{Ge}(\mathrm{Li})$ detector, $7 \mathrm{~mm}$ thick, were used. The detectors were placed approximately $10 \mathrm{~cm}$ from the centre of the neutron beam, with the depletion layer perpendicular to the incoming $\gamma$-rays.

The initial measurements with the Ortec detector were performed with an Ortec 109 preamplifier, main amplifier, biased amplifier and stretcher, and an Intertechnique 400-channel analyser. For the RCA detector, the electronics consisted of an Ortec 109 preamplifier, a Nuclear Enterprises main amplifier, biased amplifier and stretcher, and an Intertechnique 4096-channel analyser with a 2048-channel A.D.C. The resolution obtained with the last system was $5 \mathrm{keV}$ at $1 \mathrm{MeV}$ and about $12 \mathrm{keV}$ at $7 \mathrm{MeV}$. The overall stability of the system was quite good. This has been checked by recording spectra of radioactive sources before and after each run. Over a period of four days, it was found that for strong peaks situated in the upper half of the spectrum, the fluctuations in the peak positions were not greater than about 0.5 channels. This corresponds to an uncertainty in the energy calibration of less than $0.5 \mathrm{keV}$ for energies below $3 \mathrm{MeV}$. 
For good $\gamma$-ray energy determinations, the knowledge of the response function of the spectrometer is of utmost importance. The response function can be determined in two ways. One way is to use $\gamma$-ray sources with accurately known energies. This is only applicable, however, up to about $3 \mathrm{MeV}$. To extend the method to higher energies, one can, in a spectrum of neutron-capture $\gamma$-rays, make use of the reaction $Q$ value. Gamma-ray energies, summing up to the neutron binding energy, can then be used to determine the response function at higher energies. This method is not very accurate, because one relies on $Q$-values which are not known to better than $3-5 \mathrm{keV}$.

The other way to determine the response function of the system is with a precision pulse generator. The detector is then, of course, not included, and one has to assume a linear relationship between the energy dissipated in the detector and the charge appearing at the input of the preamplifier. The system has to be calibrated with accurately known energies, and then by means of the response function one can interpolate and extrapolate to all energies in the measured spectrum.

A precision pulse generator has been built, with a deviation from linearity less than $4 \times 10^{-5}$. With the pulse generator, the response function for the system was determined, and found to be slightly S-shaped. This shape could be fitted very well to a polynomial of the third degree

$$
P=\sum_{i=0}^{3} a_{i} c^{i},
$$

where $P$ stands for pulse height and $c$ for the corresponding channel number.

The pulse generator can be calibrated with $\gamma$-rays of accurately known energies, $E_{\gamma}$,

$$
E_{\gamma}=B_{0}+B_{1} P \text {. }
$$

The constants $a_{i}, B_{0}$ and $B_{1}$ are found by least-squares fitting of eqs. (1) and (2) to the measured data with a computer. Before and after a measurement, calibration was performed with the pulse generator and with radioactive sources. The average of these runs has been used in the fitting procedure. Table 4 gives the energies of the $\gamma$-rays used for calibration.

The full-energy, single and double-escape efficiency was determined with radioactive sources with known intensities, and with the $\gamma$-rays from the reactions ${ }^{32} \mathrm{Si}(\mathrm{n}, \gamma){ }^{33} \mathrm{~S}$ and ${ }^{35} \mathrm{Cl}(\mathrm{n}, \gamma){ }^{36} \mathrm{Cl}$. The intensities of the dominant lines from these two reactions were previously determined with a scintillation detector ${ }^{6}$ ) and with a magnetic Compton spectrometer ${ }^{7}$ ).

\section{Results and discussion}

Gamma rays from neutron capture in natural magnesium have been measured with the $5 \mathrm{~cm}^{3} \mathrm{Ge}(\mathrm{Li})$ detector in three runs, covering the ranges $E_{\gamma}=0-3.5 \mathrm{MeV}, 0-7.2$ $\mathrm{MeV}$ and 5-12 MeV. Non-overlapping parts of these spectra for energies up to 8.3 $\mathrm{MeV}$ are shown in fig. 1 . Above this energy no $\gamma$-ray peaks were observed.

Gamma rays from neutron capture in ${ }^{25} \mathrm{Mg}$ have been measured with the $1.6 \mathrm{~cm}^{3}$ 


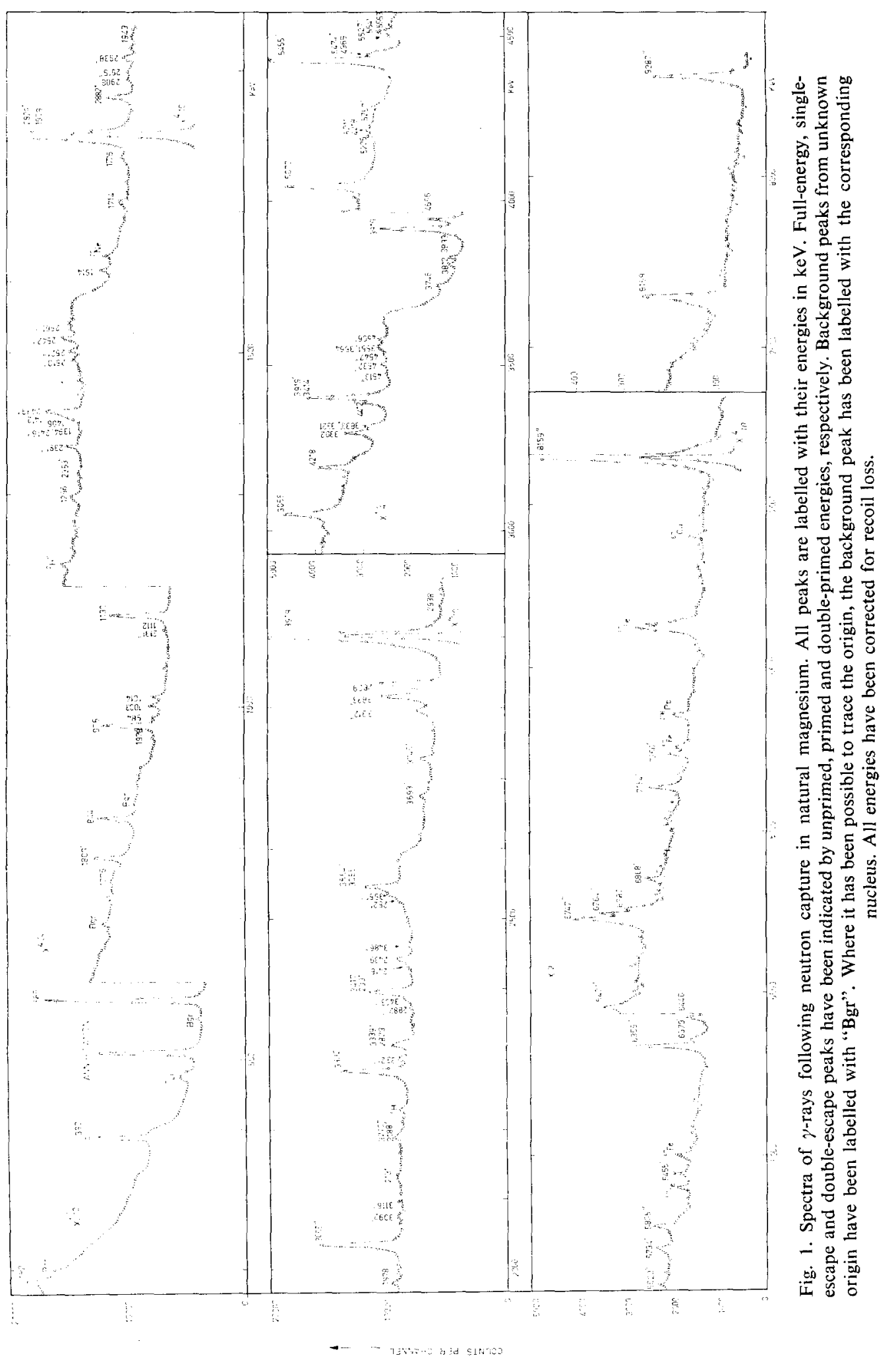









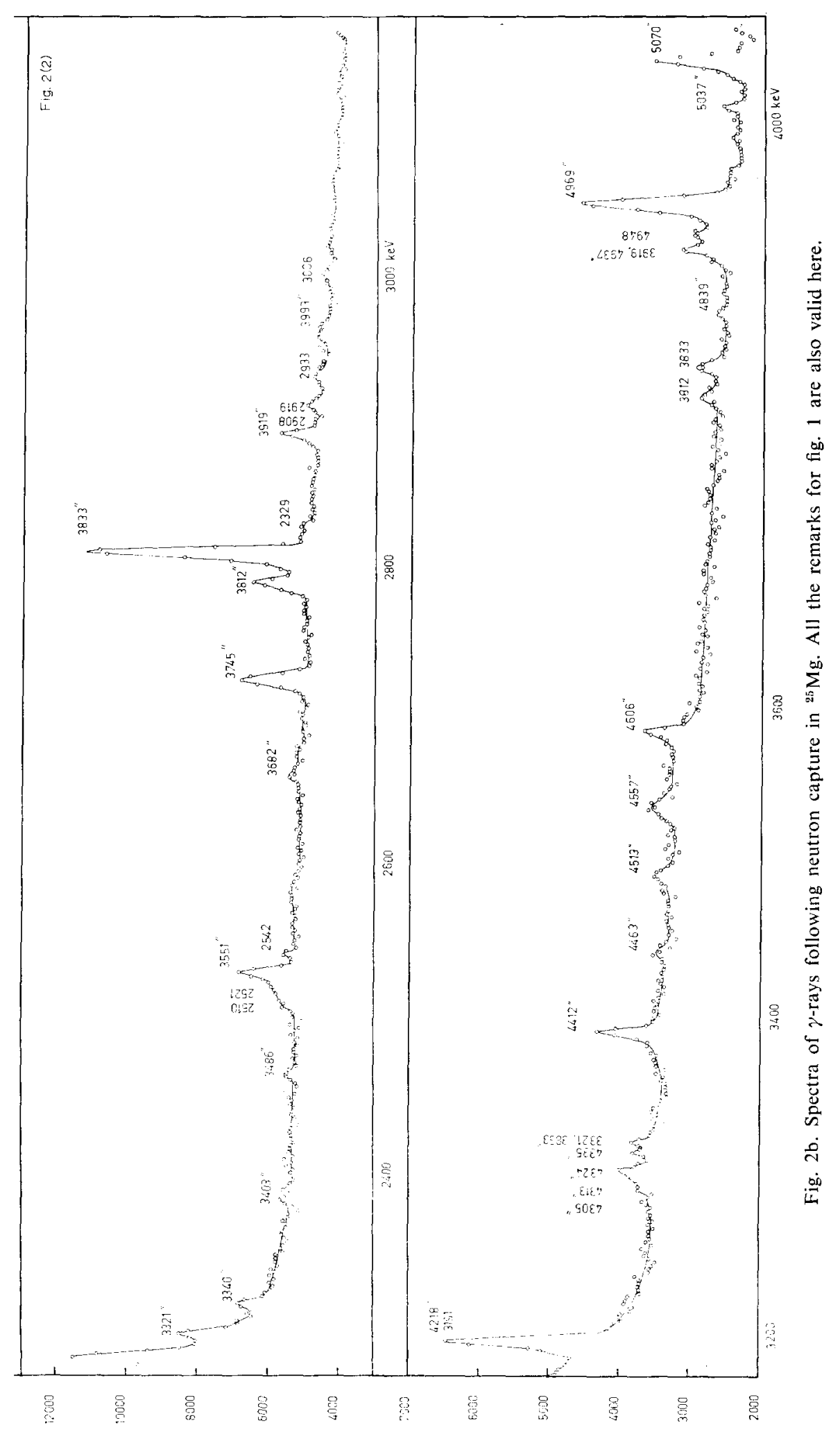


TABLE 1

Gamma rays from thermal-neutron capture in natural magnesium and in ${ }^{25} \mathrm{Mg}$. All energies are corrected for recoil loss

\begin{tabular}{|c|c|c|c|}
\hline $\begin{array}{c}E_{\gamma} \\
(\mathrm{keV})\end{array}$ & $\begin{array}{l}\text { Relative } \\
\text { intensity }\end{array}$ & $\begin{array}{c}\text { Final } \\
\mathrm{Mg} \text { isotope }\end{array}$ & $\begin{array}{l}\text { Probable } \\
\text { transition } \\
\left(E_{\mathrm{x}} \text { in } \mathrm{keV}\right)\end{array}$ \\
\hline $182.0 \pm 0.3$ & $20 \pm 5$ & \multirow{3}{*}{25} & \\
\hline $389.7 \pm 0.3$ & $420 \pm 60$ & & \multirow{2}{*}{$\begin{array}{l}\quad 975 \rightarrow 585 \\
\text { annihil. rad. }\end{array}$} \\
\hline $511.0 \pm 0.3$ & $2100 \pm 300$ & & \\
\hline $585.2 \pm 3.0$ & $2000 \pm 300$ & 25 & \multirow[t]{2}{*}{$585 \rightarrow$} \\
\hline $704.0 \pm 3.0$ & $10 \pm \quad 5$ & 26 & \\
\hline $762.4 \pm 3.0$ & $10 \pm 5$ & 26 & $4350 \rightarrow 3585$ \\
\hline $844.1 \pm 0.3$ & $140 \pm 20$ & ${ }^{27} \mathrm{Mg}\left(\beta^{-}\right)^{67} \mathrm{Al}$ & $844 \rightarrow \quad 0$ \\
\hline $974.7 \pm 0.3$ & $410 \pm 60$ & 25 & $975 \rightarrow$ \\
\hline $984.1 \pm 0.5$ & $60 \pm 10$ & 27 & $984 \rightarrow$ \\
\hline $1002.9 \pm 0.5$ & $110 \pm 15$ & 26 & $3941 \rightarrow 2938$ \\
\hline $1014.3 \pm 0.5$ & $70 \pm 10$ & ${ }^{27} \mathrm{Mg}\left(\beta^{-}\right)^{27} \mathrm{Al}$ & $1014 \rightarrow \quad 0$ \\
\hline $1097.6 \pm 1.0$ & $15 \pm \quad 5$ & 26 & \multirow[b]{2}{*}{ - } \\
\hline $1112.0 \pm 3.0$ & $15 \pm 5$ & 26 & \\
\hline $1129.5 \pm 0.3$ & $525 \pm 80$ & 26 & $2938 \rightarrow 1809$ \\
\hline $1172.6 \pm 2.0$ & $10 \pm 5$ & 26 & $5485 \rightarrow 4313$ \\
\hline $1296.1 \pm 1.0$ & $35 \pm 5$ & 26 & $8179 \rightarrow 6879$ \\
\hline $1394.1 \pm 2.0$ & $20 \pm 10$ & 26 & \multirow[t]{2}{*}{$4331 \rightarrow 2938$} \\
\hline $1405.7 \pm 2.0$ & $25 \pm 10$ & 26 & \\
\hline $1411.5 \pm 2.0$ & $75 \div 10$ & 26 & $4350 \rightarrow 2938$ \\
\hline $1613.7 \pm 1.5$ & $115 \pm 20$ & 25 & $1614 \rightarrow$ \\
\hline \multirow[t]{2}{*}{$1713.7 \div 1.0$} & $80 \pm 10$ & 25 & $4277 \rightarrow 2562$ \\
\hline & & & $3585 \rightarrow 1809$ \\
\hline $1775.8 \pm 2.0$ & $120 \pm 30$ & 26 & \multirow{2}{*}{$\begin{array}{l}6127 \rightarrow 4350 \\
7262 \rightarrow 5485\end{array}$} \\
\hline & & & \\
\hline $1808.8 \pm 0.5$ & $1060 \pm 160$ & 26 & \multirow{2}{*}{$\left.\begin{array}{cc}1809 \rightarrow & 0 \\
(1942 \rightarrow & 0\end{array}\right)$} \\
\hline $1942.5 \pm 2.0$ & $20 \pm 10$ & $(27)$ & \\
\hline $1977.6 \pm 2.0$ & $70 \div \quad 10$ & 25 & $2562 \rightarrow 585$ \\
\hline $2131.3 \pm 2.0$ & $70 \pm 15$ & 26 & $3941 \rightarrow 1809$ \\
\hline $2187.5 \pm 2.0$ & $40 \doteq 20$ & 26 & $6127 \rightarrow 3941$ \\
\hline $2352.5 \pm 1.5$ & $35 \div 10$ & \multirow[t]{3}{*}{26} & $5291 \rightarrow 2938$ \\
\hline $2390.8 \pm 1.0$ & $50 \pm 10$ & & \\
\hline $2416.0 \pm 2.0$ & $20 \pm 10$ & & \\
\hline $2438.7 \pm 1.0$ & $275 \pm 40$ & 25 & \multirow[t]{2}{*}{$3414 \rightarrow 975$} \\
\hline $2509.9 \pm 1.0$ & $40 \pm 10$ & 26 & \\
\hline $2521.4 \pm 1.5$ & $80 \div 10$ & 26 & $4331 \rightarrow 1809$ \\
\hline $2541.8 \leq 1.0$ & $60 \div 10$ & 26 & $4350 \rightarrow 1809$ \\
\hline $2561.1=1.5$ & $15 \pm 10$ & 25 & $2562 \rightarrow 0$ \\
\hline $2828.8 \pm 0.3$ & $1450 \pm 220$ & 25 & $3414 \rightarrow 585$ \\
\hline $2882.4 \div 0.3$ & $130 \pm 20$ & 27 & $C \rightarrow 3564$ \\
\hline $2908.3 \pm 1.5$ & $25 \Perp \quad 5$ & 26 & $C \rightarrow 8188$ \\
\hline $2918.5 \pm 1.5$ & $30 \pm 10$ & 26 & $C \rightarrow 8179$ \\
\hline $2937.7 \pm 1.5$ & $50 \pm 10$ & 26 & $2938 \rightarrow 0$ \\
\hline $3005.5 \pm 1.5$ & $30 \pm 10$ & 26 & \\
\hline $3054.5 \pm 0.5$ & $465 \pm 70$ & 25 & $C \rightarrow 4277$ \\
\hline $3092.0 \pm 2.0$ & $15 \pm 5$ & 26 & \\
\hline $3116.0 \div 2.0$ & $65 \pm 30$ & $(27)$ & $(3116 \rightarrow 0)$ \\
\hline $3191.0 \pm 2.0$ & $10 \pm 5$ & 26 & $7543 \rightarrow 4350$ \\
\hline
\end{tabular}


TABle 1 (continued)

\begin{tabular}{|c|c|c|c|}
\hline $\begin{array}{c}E_{\gamma} \\
(\mathrm{keV})\end{array}$ & $\begin{array}{l}\text { Relative } \\
\text { intensity }\end{array}$ & $\begin{array}{c}\text { Final } \\
\mathrm{Mg} \text { isotope }\end{array}$ & $\begin{array}{c}\text { Probable } \\
\text { transition } \\
\left(E_{\mathrm{x}} \text { in keV) }\right.\end{array}$ \\
\hline $3210.0 \pm 2.0$ & $25 \pm \quad 5$ & 26 & $7543 \rightarrow 4331$ \\
\hline $3302.0 \pm 0.5$ & $360 \pm 35$ & 25 & $4277 \rightarrow 975$ \\
\hline $3321.0 \pm 1.0$ & $40 \pm 15$ & 26 & $7262 \rightarrow 3941$ \\
\hline $3340.0 \pm 1.5$ & $25 \pm \quad 5$ & 26 & $7281 \rightarrow 3941$ \\
\hline $3403.0 \pm 2.0$ & $10 \pm \quad 5$ & 26 & \\
\hline $3414.6 \pm 1.0$ & $125 \pm 30$ & 25 & $3414 \rightarrow \quad 0$ \\
\hline $3486.0 \pm 2.0$ & $5 \pm 4$ & 26 & \\
\hline $3551.4 \pm 1.5$ & $60 \pm 10$ & 26 & $C \rightarrow 7543$ \\
\hline $3563.8 \pm 1.5$ & $170 \pm 35$ & 27 & $3564 \rightarrow \quad 0$ \\
\hline $3681.5 \pm 3.0$ & $10 \pm 5$ & 26 & $7262 \rightarrow 3585$ \\
\hline $3692.9 \pm 1.5$ & $55 \pm 10$ & 25 & $4277 \rightarrow 585$ \\
\hline $3745.8 \pm 1.5$ & $65 \pm 10$ & 26 & $\mathrm{C} \rightarrow 7350$ \\
\hline $3812.3 \pm 0.7$ & $50 \pm 10$ & 26 & $C \rightarrow 7281$ \\
\hline $3833.1 \pm 0.7$ & $240 \pm 35$ & 26 & $C \rightarrow 7262$ \\
\hline $3918.6 \pm 0.7$ & $1900 \pm 300$ & 25 & $C \rightarrow 3414$ \\
\hline $3993.2 \pm 3.0$ & $10 \pm 5$ & 26 & \\
\hline $4128.4 \pm 0.7$ & $70 \pm 10$ & 26 & $C \rightarrow 6879$ \\
\hline $4305.0 \pm 2.0$ & $4 \pm 2$ & 26 & \\
\hline $4313.0 \pm 3.0$ & $7 \pm \quad 2$ & 26 & $4313 \rightarrow 0$ \\
\hline $4324.0 \pm 2.0$ & $20 \pm 5$ & 26 & $7262 \rightarrow 2938$ \\
\hline $4336.0 \pm 2.0$ & $10 \pm 3$ & 26 & \\
\hline $4412.5 \pm 1.5$ & $45 \pm 7$ & 26 & $7350 \rightarrow 2938$ \\
\hline $4462.9 \pm 2.0$ & $6 \pm 2$ & 26 & \\
\hline $4513.2 \pm 2.0$ & $15 \pm 3$ & 26 & \\
\hline $4531.5 \pm 1.0$ & $15 \pm 3$ & & \\
\hline $4557.0 \pm 1.5$ & $20 \pm 3$ & 26 & \\
\hline $4606.2 \pm 1.0$ & $20 \pm \quad 3$ & 26 & $7543 \rightarrow 2938$ \\
\hline $4889.1 \pm 2.0$ & $4 \pm \quad 2$ & 26 & \\
\hline $4937.0 \pm 1.0$ & $25 \pm 5$ & 26 & \\
\hline $4948.0 \pm 2.0$ & $20 \pm 5$ & 26 & \\
\hline $4969.1 \pm 1.0$ & 90 土 15 & 26 & $C \rightarrow 6127$ \\
\hline $5036.8 \pm 1.5$ & $7 \pm \quad 2$ & 26 & \\
\hline $5070.2 \pm 1.5$ & $50 \pm 10$ & 26 & $6879 \rightarrow 1809$ \\
\hline $5225.0 \pm 1.5$ & $6 \pm 8$ & & \\
\hline $5240.6 \pm 2.0$ & $6 \pm 2$ & 26 & $8179 \rightarrow 2938$ \\
\hline $5256.6 \pm 3.0$ & $15 t 3$ & 26 & \\
\hline $5455.0 \pm 1.0$ & $115 \pm 15$ & 26 & $7262 \rightarrow 1809$ \\
\hline $5473.5 \pm 1.5$ & $5 \pm \quad 2$ & 26 & $7281 \rightarrow 1809$ \\
\hline $5526.5 \pm 2.0$ & $10 \pm \quad 4$ & & \\
\hline $5540.8 \pm 1.5$ & $20 \pm 5$ & 26 & $7350 \rightarrow 1809$ \\
\hline $5558.2 \pm 2.0$ & $6 \pm 2$ & 26 & \\
\hline $5622.2 \pm 1.5$ & $4 \pm 2$ & & \\
\hline $5733.6 \pm 1.5$ & $4 \pm \quad 2$ & 26 & $7543 \rightarrow 1809$ \\
\hline $5804.5 \pm 1.5$ & 15 土 3 & 26 & $C \rightarrow 5291$ \\
\hline $6358.7 \pm 1.0$ & $50 \pm 8$ & 25 & $\mathrm{C} \rightarrow 975$ \\
\hline $6379.0 \pm 1.5$ & $12 \pm 3$ & 26 & $8188 \rightarrow 1809$ \\
\hline $6446.3 \pm 1.5$ & $20 \pm 4$ & 27 & $C \rightarrow \quad 0$ \\
\hline $6470.2 \pm 1.5$ & $8 \pm 2$ & & \\
\hline
\end{tabular}


TABLE 1 (continued)

\begin{tabular}{|c|c|c|c|}
\hline $\begin{array}{c}E_{\gamma} \\
(\mathrm{keV})\end{array}$ & $\begin{array}{l}\text { Relative } \\
\text { intensity }\end{array}$ & $\begin{array}{c}\text { Final } \\
\mathrm{Mg} \text { isotope }\end{array}$ & $\begin{array}{c}\text { Probable } \\
\text { transition } \\
\left(E_{\mathrm{x}} \text { in } \mathrm{keV}\right)\end{array}$ \\
\hline $6746.7 \pm 1.0$ & $40 \pm 5$ & $\left\{\begin{array}{l}25 \\
26\end{array}\right.$ & $\begin{array}{l}\mathrm{C} \rightarrow 585 \\
\mathrm{C} \rightarrow 4350\end{array}$ \\
\hline $6763.7+1.5$ & $12 ! 2$ & 26 & $C \rightarrow 4331$ \\
\hline $6781.8 \pm 3.0$ & $3 \pm 1$ & 26 & $C \rightarrow 4313$ \\
\hline $6867.7+2.0$ & $2-1$ & & \\
\hline $7154.3 \pm 1.5$ & $5 \pm 2$ & 26 & $C \rightarrow 3941$ \\
\hline $7255.7+2.0$ & $5+2$ & 26 & \\
\hline $8159.0+1.0$ & $180 \pm 25$ & 26 & $C \rightarrow 2938$ \\
\hline $9286.9 \pm 1.0$ & $40 \pm 5$ & 26 & $C \rightarrow 1809$ \\
\hline
\end{tabular}

The errors in the energies are mainly due to errors made in reading-off peak positions. The errors in the intensities are partly due to statistics, partly due to uncertainties in the efficiency curves for the detector. The efficiency curves are believed to be true to within $15 \%$. The intensities given all refer to capture in natural magnesium.

$\mathrm{Ge}(\mathrm{Li})$ detector and a 400 -channel analyser. Four of these spectra, covering the range $E_{\gamma}=0-5 \mathrm{MeV}$, are shown in fig. 2 .

The peaks are labelled with unprimed, primed and double-primed energies in $\mathrm{keV}$, indicating full-energy, single and double-escape peaks. All energies were corrected for recoil loss. Where it has been possible to trace the origin of a background peak, the peak has been labelled with the corresponding final nucleus, otherwise only with "Bgr".

The background radiation originates from neutron capture in the iron and lead shielding around neighbouring neutron diffraction experiments, from neutron capture in a copper monochromator in one of the diffraction experiments and from neutron capture in the teflon target holder. In order to get a better understanding of the background radiation, separate measurements of $\gamma$-rays from neutron capture in iron, copper and teflon have been performed.

In table 1 , all observed $\gamma$-rays which can be assigned to capture in one of the three magnesium isotopes or to the ${ }^{27} \mathrm{Mg}\left(\beta^{-}\right)^{27} \mathrm{Al}$ decay, are listed. In the determination of energies, three types of errors have been considered. These are, errors made in readingoff peak positions, errors due to the differential non-linearity of the analyser and errors made in calibrating spectra. Only the first type of errors has been found to be of importance for this investigation. This conclusion was based on several considerations. For strong lines in the measured spectra, where both full-energy and double-escape peaks were seen, one finds the correct energy difference, $1022 \mathrm{keV}$. One can compare the $Q$-values determined in the present paper, with those calculated from the 1964 mass table ${ }^{14}$ ) and with recent determinations of $Q$-values from the reactions ${ }^{56} \mathrm{Fe}(\mathrm{n}, \gamma){ }^{57} \mathrm{Fe},{ }^{63} \mathrm{Cu}(\mathrm{n}, \gamma){ }^{64} \mathrm{Cu}$ and ${ }^{207} \mathrm{~Pb}(\mathrm{n}, \gamma){ }^{208} \mathrm{~Pb}\left(\right.$ ref. $\left.\left.{ }^{15}\right)\right)$. The comparison is shown in table 3 . The agreement is very good. Also, one finds good internal agree- 
TABLE 2

$Q$-values for the reactions ${ }^{24} \mathrm{Mg}(\mathrm{n}, \gamma)^{25} \mathrm{Mg},{ }^{25} \mathrm{Mg}(\mathrm{n}, \gamma)^{26} \mathrm{Mg}$ and ${ }^{26} \mathrm{Mg}(\mathrm{n}, \gamma)^{27} \mathrm{Mg}$ found by calculating the weighted average of the sums of $\gamma$-ray energies in cascades between capturing state and ground state in the three isotopes ${ }^{25} \mathrm{Mg},{ }^{26} \mathrm{Mg}$ and ${ }^{27} \mathrm{Mg}$

internal error 0.5

$$
\begin{array}{ll}
{ }^{24} \mathrm{Mg}(\mathrm{n}, \gamma)^{25} \mathrm{Mg} & \\
6746.7+585.2 & =7331.9 \pm 1.0 \\
6358.7+974.7 & =7333.4 \pm 1.0 \\
3918.6+3414.6 & =7333.2 \pm 0.8 \\
3918.6+2828.8+585.2 & =7332.6 \pm 1.3 \\
3918.6+2438.7+974.7 & =7332.0 \pm 1.2 \\
3054.5+3692.9+585.2 & =7332.6 \pm 1.6 \\
3054.5+3302.0+974.7 & =7331.2 \pm 0.8 \\
\text { weighted average } & -7332.8
\end{array}
$$$$
6358.7+974.7=7333.4 \pm 1.0
$$$$
3918.6+2828.8+585.2=7332.6 \pm 1.3
$$$$
3054.5+3692.9+585.2=7332.6 \pm 1.6
$$

weighted average

external error 0.4

Adopted value: $Q=7332.8 \pm 0.5 \mathrm{keV}$.

All energies are in $\mathrm{keV}$. The internal and external errors have been calculated in every case, and it was always found that the internal errors are bigger than the external errors. This should indicate that the estimated errors in the measured energies are too big. The internal errors have been chosen as errors in the $Q$-value.

$\begin{array}{ll} & \begin{array}{l}{ }^{25} \mathrm{Mg}(\mathrm{n}, \gamma)^{26} \mathrm{Mg} \\ \text { Cascades to }{ }^{26} \mathrm{Mg}(1)\end{array} \\ 9286.9 & =9286.9 \pm 1.0 \\ 8159.0+1129.5 & =9288.5 \pm 1.0 \\ 7154.3+2131.3 & =9285.6 \pm 2.5 \\ 6763.7+2521.4 & =9285.1 \pm 2.1 \\ 6746.7+2541.8 & =9288.5 \pm 2.2 \\ 4218.4+5070.2 & =9288.6 \pm 1.7 \\ 3833.1+5455.0 & =9288.1 \pm 1.2 \\ 3812.3+5473.5 & =9285.8 \pm 1.7 \\ 3745.8+5540.8 & =9286.6 \pm 2.1 \\ 3551.4+5733.6 & =9285.0 \pm 2.1 \\ 2908.3+6379.0 & =9287.3 \pm 2.1 \\ \text { weighted average } & =9287.3\end{array}$

internal error 0.5

external error 0.4

Energy difference between the capturing state and the first excited state is $9287.3 \pm 0.5 \mathrm{keV}$.

$\begin{array}{ll}8159.0 & =8159.0 \pm 1.0 \\ 7154.3+1002.9 & =8157.0 \pm 1.6 \\ 6763.7+1394.1 & =8157.8 \pm 2.5 \\ 6746.7+1411.5 & =8158.2 \pm 2.8 \\ 5804.5+2352.5 & =8157.0 \pm 2.1 \\ 4969.1+2187.5+1002.9 & =8159.5 \pm 2.3 \\ 3833.1+4324.0 & =8157.1 \pm 2.1 \\ 3833.1+3321.0+1002.9 & =8157.0 \pm 1.3 \\ 3812.3+3340.0+1002.9 & =8155.2 \pm 1.7 \\ 3745.8+4412.5 & =8158.3 \pm 2.1 \\ 3551.4+4606.2 & =8157.6 \pm 1.8 \\ 2918.5+5240.6 & =8159.1 \pm 2.5 \\ \text { weighted average } & =8157.8\end{array}$

internal error 0.5

external error 0.4

Energy difference between the capturing state and the second excited state is $8157.8 \pm 0.5 \mathrm{keV}$. 
$9287.3+1808.8$

$8157.8+2937.7$

$8157.8+1808.8+1129.5$

$4313.0+6781.8$

weighted average
$=11096.1 \pm 0.7$

$=11095.5-1.6$

$=11096.1 \pm 0.8$

$=11094.8 \pm 4.2$

11096.0

internal error 0.5

external error 0.1

Adopted value: $Q=11096.0 \pm 0.5 \mathrm{keV}$



All values are in keV.

ment for the $Q$-values determined from cascades between the capturing state and the ground state.

The intensities given in table 1 refer to capture in natural magnesium. Intensities of lines found from capture in ${ }^{25} \mathrm{Mg}$ have been normalized to capture in natural magnesium. The errors in the intensities are partly due to statistics, and partly due to the uncertainties in the efficiency curves for the detectors. These curves are believed to be true to within $15 \%$.

The decay schemes of ${ }^{26} \mathrm{Mg}$ and ${ }^{27} \mathrm{Mg}$ are shown in fig. 3 and those of ${ }^{25} \mathrm{Mg}$ and the mirror nucleus, ${ }^{25} \mathrm{Al}$, in fig. 4 . The decay scheme of ${ }^{25} \mathrm{Al}$ is from ref. ${ }^{3}$ ), and only the part relevant to the decay in ${ }^{25} \mathrm{Mg}$ is shown. The given intensities in the three $\mathrm{Mg}$ isotopes are normalized such that the sum of the intensities of primary $\gamma$-rays equals 100. The same has been done for the decay of the various levels.

In fitting $\gamma$-rays into the level schemes of the three isotopes, it was required that the sum of the energies of $\gamma$-rays in a cascade going from capturing state to ground state, should be equal to the $Q$-value within $3 \mathrm{keV}$. In this way, most of the $\gamma$-rays in table 1 could be fitted between known levels. Comparison of the sum of the intensities of the 
lines feeding a level with that of the lines by which the same level is de-excited gives a check on the consistency of the three decay schemes. For all levels in ${ }^{25} \mathrm{Mg}$ quite good agreement is found. In ${ }^{26} \mathrm{Mg}$, the intensity sum of $\gamma$-rays feeding the two lowest levels is $20 \%$ smaller than that of the $\gamma$-rays by which they are de-excited. This indicates that weak $\gamma$-rays leading to these two levels may have been missed. For the rest of the levels in ${ }^{26} \mathrm{Mg}$ the consistency is reasonable.
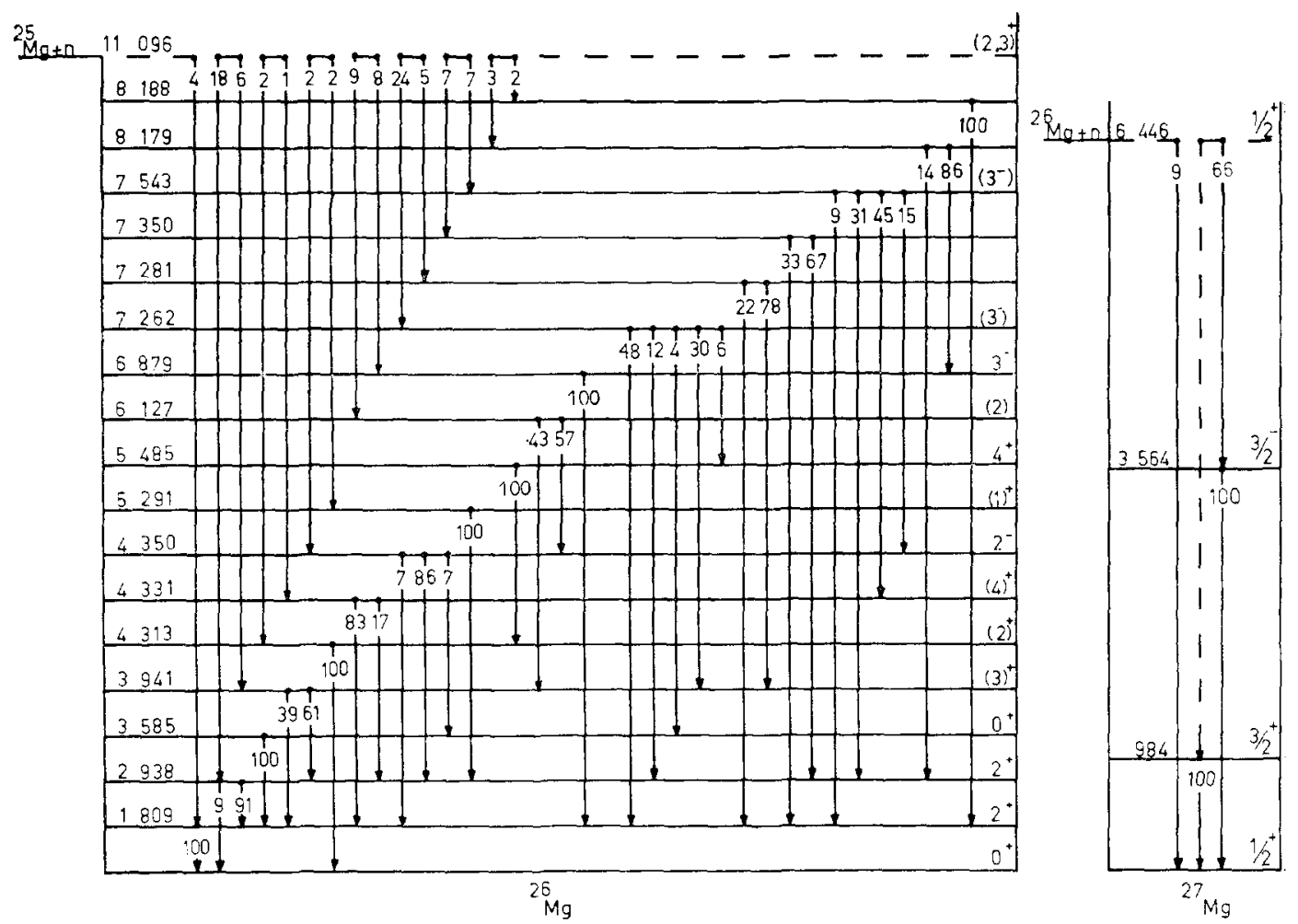

Fig. 3. Decay schemes of ${ }^{26} \mathrm{Mg}$ and ${ }^{27} \mathrm{Mg}$. The level energies are given in $\mathrm{keV}$. The intensities are normalized for each nucleus such that the intensity sum of all primary $\gamma$-rays equals 100 . Spins and parities are from refs. ${ }^{3,4,15}$ ).

For the cascade $\mathrm{C} \rightarrow 3564 \rightarrow 0$ in ${ }^{27} \mathrm{Mg}$, the consistency is good. The intensity sum of ground-state transitions in ${ }^{27} \mathrm{Mg}$ and the intensity sum of ground-state transitions in the decay product ${ }^{27} \mathrm{Al}$ are approximately equal. This means that the most intense lines in the decay scheme of ${ }^{27} \mathrm{Mg}$ have been found.

Sum-coincidence measurements performed with two NaI detectors, show that the only possible two-step cascades in ${ }^{26} \mathrm{Mg}$ are those going via the first, second and fifth level.

In ${ }^{25} \mathrm{Mg}$, four new transitions have been found. These are transitions between the following levels (in $\mathrm{keV}$ ) $4277 \rightarrow 585,4277 \rightarrow 2562,2562 \rightarrow 0,2562 \rightarrow 585$. 
Most of the transitions found in ${ }^{26} \mathrm{Mg}$ have not been observed before. Four highenergy lines with $E_{\gamma}=11.09 \pm 0.03,10.08 \pm 0.02,8.93 \pm 0.02,8.55 \pm 0.02 \mathrm{MeV}$ found by Campion and Bartholomew ${ }^{1}$ ), were not seen in the present investigation. They were not observed either by Groshev ${ }^{2}$ ). Since the detector used in the present investigation is small, it is not strange that the weak $11.09 \mathrm{MeV}$ line is not seen. But the three other lines should have been observed. Either the intensities of these lines are smaller than those given in ref. ${ }^{1}$ ), or the lines originate from impurities.

In ${ }^{27} \mathrm{Mg}$, one new cascade was found, viz. $\mathrm{C} \rightarrow 3564 \rightarrow 0$.


Fig. 4. Decay schemes of ${ }^{25} \mathrm{Mg}$ and ${ }^{25} \mathrm{Al}$. The intensities of $\gamma$-rays in ${ }^{25} \mathrm{Mg}$ are normalized such that the intensity sum of primary $\gamma$-rays equals 100 . The decay scheme of ${ }^{25} \mathrm{Al}$ and spins and parities are from ref. ${ }^{3}$ ).

Table 2 lists the cascades used for calculating the neutron binding energies for the three isotopes. For ${ }^{25} \mathrm{Mg}$ and ${ }^{27} \mathrm{Mg}$, this has been done in a straightforward way. In ${ }^{26} \mathrm{Mg}$, nearly all $\gamma$-ray cascades are going via the first and second excited state. Here, the weighted averaged energy differences between the capturing state and these two levels have first been calculated. In calculating the errors in the $Q$-values, it was always found that the internal errors were bigger than the external errors. This indicates that the estimated errors in the energies in table 1 are too big. The results of these cal- 
culations are

$$
\begin{array}{ll}
{ }^{24} \mathrm{Mg}(\mathrm{n}, \gamma){ }^{25} \mathrm{Mg}: & Q=7332.8 \pm 0.4 \mathrm{keV}, \\
{ }^{25} \mathrm{Mg}(\mathrm{n}, \gamma){ }^{26} \mathrm{Mg}: & Q=11096.0 \pm 0.5 \mathrm{keV}, \\
{ }^{26} \mathrm{Mg}(\mathrm{n}, \gamma){ }^{27} \mathrm{Mg}: & Q=6446.3 \pm 0.9 \mathrm{keV} .
\end{array}
$$

Also $Q$-values for neutron capture in ${ }^{56} \mathrm{Fe},{ }^{63} \mathrm{Cu}$ and ${ }^{207} \mathrm{~Pb}$ were determined. These $Q$-values are listed in table 3 . For comparison, the $Q$-values calculated from the 1964 mass table ${ }^{14}$ ), and $Q$-values for capture in ${ }^{56} \mathrm{Fe},{ }^{63} \mathrm{Cu}$ and ${ }^{207} \mathrm{~Pb}$ recently determined ${ }^{15}$ ), are also listed. The agreement is quite good.

The thermal-neutron capture cross sections for the three isotopes and for natural magnesium, are measured to be $34 \pm 10 \mathrm{mb}, 280 \pm 90 \mathrm{mb}, 27 \pm 5 \mathrm{mb}$ and $63 \pm 3 \mathrm{mb}$, respectively ${ }^{3}$ ).

From these values and the abundances, one should expect for capture in natural magnesium approximately equal capture contributions in ${ }^{24} \mathrm{Mg}$ an ${ }^{25} \mathrm{Mg}$, viz. $45-50 \%$ in each, and approximately $5 \%$ capture in ${ }^{26} \mathrm{Mg}$. From the present investigation it follows, when one uses the intensity sum of primary $\gamma$-rays in ${ }^{25} \mathrm{Mg}$ and of ground-state $\gamma$-rays in ${ }^{26} \mathrm{Mg}$ and ${ }^{27} \mathrm{Mg}$, that the isotopic capture cross sections are

$$
\begin{aligned}
& { }^{24} \mathrm{Mg}(\mathrm{n}, \gamma){ }^{25} \mathrm{Mg}=52 \pm 13 \mathrm{mb}, \\
& { }^{25} \mathrm{Mg}(\mathrm{n}, \gamma){ }^{26} \mathrm{Mg}=181 \pm 50 \mathrm{mb} \text {, } \\
& { }^{26} \mathrm{Mg}(\mathrm{n}, \gamma){ }^{27} \mathrm{Mg}=34 \pm 10 \mathrm{mb} \text {. }
\end{aligned}
$$

Excitation energies of several levels in ${ }^{25} \mathrm{Mg},{ }^{26} \mathrm{Mg},{ }^{27} \mathrm{Mg}$ and ${ }^{27} \mathrm{Al}$ can be determined with a precision exceeding that obtained with the $(d, p)$ reaction $\left.{ }^{3}\right)$. These energies are presented in table 5 .

Since ${ }^{25} \mathrm{Mg}$ and ${ }^{25} \mathrm{Al}$ are mirror nuclei, one should expect a close relationship between the level structures of the two nuclei and between the decay pattern associated with mirror levels. It is therefore of some value to compare the decay scheme of ${ }^{25} \mathrm{Mg}$ found in the present investigation with the relevant part of the decay scheme of ${ }^{25} \mathrm{Al}$ found in ref. ${ }^{3}$ ). The two decay schemes are shown in fig. 4 . One can see that the branching ratios for the two negative parity states in ${ }^{25} \mathrm{Mg}$ are in good agreement with those for the corresponding states in ${ }^{25} \mathrm{Al}$. Poor agreement is found for the branching ratios for the two mirror levels at $E_{\mathrm{x}}=2.5 \mathrm{MeV}$. In ${ }^{25} \mathrm{Al}$ this level has transitions to the first and second excited state and a very weak transition to the ground state. The mirror level in ${ }^{25} \mathrm{Mg}$ has only transitions to the ground state and the first excited state. The main part of the decay of these two mirror levels goes to the first excited states. The disagreement in the decay of these levels may perhaps partly be ascribed to the fact that transitions between intrinsic states in ${ }^{25} \mathrm{Mg}$ are due to a single neutron jump, while those in ${ }^{25} \mathrm{Al}$ are due to a single proton jump.

Theoretical investigations of the level structures of ${ }^{25} \mathrm{Mg}$ and ${ }^{25} \mathrm{Al}$ (refs. ${ }^{18,19}$ )) show that it is possible to construct rotational bands based on the mirror levels at 
$E_{\mathrm{x}}=2.5 \mathrm{MeV}$. These rotational bands may be strongly perturbed by the overlapping negative parity rotational band based on the $\frac{3}{2}^{-}$levels shown in fig. 4 . This perturba-

TABLE 4

Energies of $\gamma$ rays used for calibration

\begin{tabular}{ccc} 
Isotope & \multicolumn{1}{c}{$E_{\gamma}(\mathrm{keV})$} & Ref. \\
\hline${ }^{57} \mathrm{Co}$ & $122.05 \pm 0.05$ & $\left.{ }^{8}\right)$ \\
ann. rad. & $511.006 \pm 0.002$ & $\left.{ }^{9}\right)$ \\
${ }^{137} \mathrm{Cs}$ & $661.595 \pm 0.076$ & $\left.{ }^{10}\right)$ \\
${ }^{54} \mathrm{Mn}$ & $834.84 \pm 0.07$ & $\left.{ }^{11}\right)$ \\
${ }^{88} \mathrm{Y}$ & $898.01 \pm \mathbf{0 . 0 7}$ & $\left.{ }^{11}\right)$ \\
${ }^{60} \mathrm{Co}$ & $1173.226 \pm 0.040$ & $12)$ \\
${ }^{22} \mathrm{Na}$ & $1274.52 \pm 0.07$ & $\left.{ }^{11}\right)$ \\
${ }^{60} \mathrm{Co}$ & $1332.483 \pm 0.046$ & $\left.{ }^{22}\right)$ \\
${ }^{24} \mathrm{Na}$ & $1368.526 \pm 0.044$ & $\left.{ }^{12}\right)$ \\
${ }^{88} \mathrm{Y}$ & $1836.08 \pm \mathbf{0 . 0 7}$ & $\left.{ }^{11}\right)$ \\
${ }^{1} \mathrm{H}(\mathrm{n}, \gamma)^{2} \mathrm{H}$ & $2224.5 \quad \pm 0.2$ & $\left.{ }^{13}\right)$ \\
${ }^{24} \mathrm{Na}$ & $2753.92 \pm 0.12$ & $\left.{ }^{8}\right)$ \\
\hline
\end{tabular}

TABLE 5

Excitation energies deduced from the present investigation

\begin{tabular}{cc}
${ }^{25} \mathrm{Mg}$ & ${ }^{26} \mathrm{Mg}$ \\
\hline $585.2 \pm 0.3$ & $1808.8 \pm 0.5$ \\
$974.7 \pm 0.3$ & $2938.0 \pm 0.3$ \\
$(1613.7 \pm 1.5)$ & $3584.7 \pm 2.0$ \\
$2561.8 \pm 1.5$ & $3940.5 \pm 0.5$ \\
$3414.0 \pm 0.5$ & $4313.0 \pm 1.5$ \\
$4277.4 \pm 1.0$ & $4331.0 \pm 1.0$ \\
& $4350.1 \pm 0.5$ \\
$27 \mathrm{Mg}$ & $5290.5 \pm 0.5$ \\
\hline $984.1 \pm 0.5$ & $5485.0 \pm 2.0$ \\
$3563.8 \pm 1.5$ & $6127.2 \pm 0.8$ \\
& $6879.1 \pm 1.5$ \\
$27 \mathrm{Al}$ & $7262.4 \pm 1.5$ \\
\hline $844.1 \pm 0.3$ & $7281.4 \pm 0.5$ \\
$1014.3 \pm 0.5$ & $7350.1 \pm 0.6$ \\
\hline
\end{tabular}

All energies are given in $\mathrm{keV}$.

tion will also affect the decay of the levels at $E_{\mathrm{x}}=2.5 \mathrm{MeV}$ and can perhaps partly explain the observed difference. In ${ }^{25} \mathrm{Mg}$ this level has a pure E2 transition to the ground state and a pure Ml transition to the first excited state. The $\gamma_{0} / \gamma_{1}$ intensity 
ratio is 0.23 , about 20 times larger than what one would expect from the Weisskopf estimates, showing that the E2 transition is collectively enhanced.

From the results of the present investigation it was not possible to establish a transition to the level at $E_{\mathrm{x}}=1.6 \mathrm{MeV}$. However, a $\gamma$-ray with energy $1614 \mathrm{keV}$ was found and interpreted as the decay of this level to the ground state.

The branching ratios for the two mirror levels around $0.95 \mathrm{MeV}$ are in agreement with each other.

The transitions found in ${ }^{26} \mathrm{Mg}$ agree with the spin and parity assignments made by Hinds, Marchant and Middleton ${ }^{5}$ ), except for the $4^{+}$assignment to the lowest component of the triplet at $E_{\mathrm{x}}=4.3 \mathrm{MeV}$. In the present investigation a $\gamma$-ray with energy $4313 \mathrm{keV}$ was found and interpreted as the ground-state transition from this level. Therefore this level cannot have $J^{\pi}$ equal to $4^{+}$as proposed, but $1^{+}$or $2^{+}$. The level is fed from a $4^{+}$level at $E_{\mathrm{x}}=5485 \mathrm{keV}$ and also weakly fed from the capturing state. Therefore the $E_{\mathrm{x}}=4313 \mathrm{keV}$ level may have $J^{\pi}=2^{+}$. The middle component of the triplet was also proposed to have $J^{\pi}=4^{+}$, and this is not in contradiction with the present results.

The levels with excitation energies higher than $6.9 \mathrm{MeV}$ involved in the present investigation are not well resolved in the $(d, p)$ reaction $\left.{ }^{4}\right)$. Groups of levels in this region show $l_{n}=1$ stripping patterns. One may therefore assume that most of the primary $\gamma$-rays feeding these levels have $\mathrm{E} 1$ character. This explains the large intensity of the $E_{\gamma}=3833 \mathrm{keV}$ transition feeding the $E_{\mathrm{x}}=7262 \mathrm{keV}$ level. This level decays to the $J^{\pi}=4^{+}, E_{\mathrm{x}}=5485 \mathrm{keV}$ level, the $3^{+}, E_{\mathrm{x}}=3941 \mathrm{keV}$ level, the two $2^{+}$levels at $E_{\mathrm{x}}=2938 \mathrm{keV}$ and $1809 \mathrm{keV}$ and, weakly, to the $0^{+}$state at $E_{\mathrm{x}}=3585 \mathrm{keV}$. The only possible assignment for the $E_{\mathrm{x}}=7262 \mathrm{keV}$ level seems to be $J^{\pi}=3^{-}$. Using similar arguments one can make tentative spin and parity assignments of $J^{\pi}=(2,3)^{-}$ to the $E_{\mathrm{x}}=7281 \mathrm{keV}$ level, $(1,2,3)^{-}$to the $E_{\mathrm{x}}=7350 \mathrm{keV}$ level and $3^{-}$to the $E_{\mathrm{x}}=7543 \mathrm{keV}$ level.

For the doublet at $8.2 \mathrm{MeV}$ both levels may have odd parity. The lowest level in the doublet has a transition to the $J^{\pi}=3^{-}$level at $E_{\mathrm{x}}=6879 \mathrm{keV}$ and a weak transition to the $J^{\pi}=2^{+}$level at $E_{\mathrm{x}}=2938 \mathrm{keV}$. The low-energy transition to the $3^{-}$level may be a strongly enhanced M1 transition, and the lower level in the doublet may therefore have odd parity with spin 2 or 3 . The other member of the doublet may then have odd parity with spin 1,2 or 3 .

The strong cascade found in ${ }^{27} \mathrm{Mg}$ going through the ${ }^{\frac{3}{2}}{ }^{-}$level at $E_{\mathrm{x}}=3564 \mathrm{keV}$ (ref. $\left.{ }^{16}\right)$ ), is in good agreement with the odd-parity assignment to this level.

An attempt was made to compare the reduced intensities of primary $\gamma$-rays feeding levels with $l_{n}=1$, with the reduced widths for the same levels in the $(\mathrm{d}, \mathrm{p})$ reaction. Such a comparison can give information about the capture mechanism.

If the capture process is dominated by direct capture, one expects to find correlation between reduced $\mathrm{E} 1$ strengths and the reduced $(\mathrm{d}, \mathrm{p})$ widths. This is the case for capture in ${ }^{24} \mathrm{Mg}$ and ${ }^{26} \mathrm{Mg}$. The decay of the capturing states is here dominated by E1 transitions with strengths exceeding the average $E 1$ strength in $(n, \gamma)$ reactions 
found by Bartholomew ${ }^{17}$ ). There are strong correlations between the reduced intensities of the primary E1 transitions and the $(d, p)$ widths of the levels they are feeding. For these two nuclei direct capture dominates.

In ${ }^{26} \mathrm{Mg}$ only one strong primary E1 transition appears with a strength approximately equal to the average E1 strength. The other primary $\gamma$-rays are weaker E1 transitions or relatively strong M1 transitions. Comparisons between reduced $\gamma$-ray intensities and reduced (d, p) widths could be made only for one level, viz. the level at $E_{\mathrm{x}}=6879 \mathrm{keV}$. For this level the (d, p) width is large, while the El strength is small. This fact and the many weak primary $\gamma$-rays suggest that capture in ${ }^{25} \mathrm{Mg}$ mainly proceeds via the compound nuclear reaction or through doorway states.

It is a pleasure to thank Professor P. M. Endt for his encouragement and great interest in this work, and for his critical reading of the manuscript. It is also a pleasure to thank R. J. S. Harry for the use of his $\mathrm{Ge}(\mathrm{Li})$ detector and 4096 channel analyser.

This work has been sponsored by the Foundation for Fundamental Research on Matter (F.O.M.).

\section{References}

1) P. J. Campion and G. A. Bartholomew, Can. J. Phys. 35 (1957) 1365

2) L. V. Groshev, V. N. Lutsenko, A. M. Demidov and V. I. Pelekhov, Atlas of $\gamma$-ray spectra from radiative capture of thermal neutrons (Pergamon, London, 1959)

3) P. M. Endt and C. van der Leun, Nuclear Physics 34 (1962) 1

4) B. Cujec, Phys. Rev. 136 (1964) B1305

5) S. Hinds, H. Marchant and R. Middleton, Nuclear Physics 67 (1965) 257

6) G. van Middelkoop and H. Gruppelaar, Nuclear Physics 80 (1966) 321

7) L. V. Groshev, A. M. Demidov and V. N. Lutsenko, Izv. Akad. Nauk (ser. fiz.) 24 (1960) 833

8) G. T. Ewan and A. J. Tavendale, Can. J. Phys. 42 (1964) 2286

9) E. R. Cohen and J. W. M. Dumond, Revs. Mod. Phys. 37 (1965) 537

10) R. L. Graham, Nucl. Instr. 9 (1960) 245

11) W. W. Black and R. L. Heath, Nuclear Physics A90 (1967) 650

12) G. Murray, R. L. Graham and J. S. Geiger, Nuclear Physics 63 (1965) 353

13) J. W. Knowles, Can. J. Phys. 40 (1962) 257

14) J. H. E. Mattauch, W. Thiele and A. H. Wapstra, Nuclear Physics 67 (1965) 32

15) G. van Middelkoop, Nuclear Physics A97 (1967) 209

16) J. M. Lacambra, D. R. Tilley and N. R. Roberson, Nuclear Physics A92 (1967) 30

17) G. A. Bartholomew, Ann. Rev. Nucl. Sci. 11 (1961) 259

18) R. K. Sheline and R. A. Harlan, Nuclear Physics 29 (1962) 177

19) A. E. Litherland, H. McManus, E. B. Paul, D. A. Bromley and H. E. Gove, Can. J. Phys. 36 (1958) 378 